\title{
SIGNAL-TO-NOISE RATIO AND ASTRONOMICAL FOURIER TRANSFORM SPECTROSCOPY
}

\author{
J.P. Maillard \\ Institut d'Astrophysique de Paris \\ 98bis Bld Arago, 75014 Paris (France)
}

\begin{abstract}
The multiplex properties of the Fourier Transform Spectrometer (FTS) can be considered as disadvantageous with modern detectors and large telescopes, the dominant noise source being no longer in most applications the detector noise. Nevertheless, a FTS offers a gain in information and other instrumental features remain: flexibility in choosing resolving power up to very high values, large throughput, essential in high-resolution spectroscopy with large telescopes, metrologic accuracy, automatic substraction of parasitic background. The signal-to-noise ratio in spectra can also be improved: by limiting the bandwidth with cold filters or even cold dispersers, by matching the instrument to low background foreoptics and high-image quality telescopes. The association with array detectors provides the solution for the FTS to regain its full multiplex advantage.
\end{abstract}

\section{INTRODUCTION}

FTS has gained the rank of high-performance spectroscopic tool for twenty years now. This instrument has largely contributed to open the new field of infrared spectroscopy by giving access to the high-resolution study of new transitions of atoms and molecules in laboratory, and to the infrared spectrum of many astronomical sources. Since that time, FTS have remained without any serious competitors in infrared laboratory spectroscopy - except in the domain of ultra high-resolution where laser techniques are dominating. The situation is different in astronomy. FTS did not become an universal instrument in the observatories. The energy from the astronomical sources is weak, the observing time limited, which put many objects out of reach of deep spectroscopic investigations. When attempted, the signal-to-noise ratio (SNR) which can be reached becomes a fundamental consideration and determines the choice of the instrumentation. However, the harsh telescope environment for any instrument imposes very often the choice and leads to prefer simpler devices, even if they are of limited capabilities. Consequently, few FTS are in operation behind ground-based telescopes. Nevertheless, an extraordinary quantity of results, which could not have been obtained by any other methods, were recorded in solar, stellar and planetary spectroscopy. The last review to date showing the variety of scientific applications was made by Ridgway and Brault (1984). An update review by de Bergh (1987) is limited to the solar system only. 
The present symposium gives the opportunity to focus on the SNR aspect of the method, to the light of modern detectors developments and then to examine its future.

\section{THE MULTIPLEX GAIN}

FT spectroscopy belongs to the class of the multiplex methods. Thirty years ago, P. Felgett pointed out that a dramatic gain in SNR, which was called the multiplex gain, could be obtained using such a method instead of sequential techniques, only known way at that time, and for many years, of entering in the era of the photoelectric detectors. Progress in that field have modified this prophetic view. A reexamination is made in Table I through the comparisons of SNR between the three classical classes of spectroscopic systems : multiplex, multichannel, sequential.

TABLE I

per spectral element multiplex

multichannel sequential

\begin{tabular}{llll} 
integration time & \multicolumn{1}{c}{$T$} & \multicolumn{1}{c}{$T$} & \multicolumn{1}{c}{$T / M$} \\
flux & $w d \sigma T$ & $w d \sigma T$ & $w d \sigma T / M$ \\
detector noise (I) & NEP $\sqrt{T}$ & NEP $\sqrt{T}$ & NEP $\sqrt{T / M}$ \\
source noise (II) & $\sqrt{w \Delta \sigma T}$ & $\sqrt{w \Delta \sigma T}$ & $\sqrt{w \Delta \sigma T / M}$ \\
backgr. noise (III) & $\sqrt{B \Delta \sigma T}$ & $\sqrt{B \Delta \sigma T}$ & $\sqrt{B \Delta \sigma T / M}$ \\
\hline SNR (I) & $w d \sigma \sqrt{T} / \mathrm{NEP}$ & $w d \sigma \sqrt{T} / \mathrm{NEP}$ & $w d \sigma \sqrt{T} / \mathrm{NEP} \times M^{-1 / 2}$ \\
SNR (II) & $\sqrt{w d \sigma T} \times M^{-1 / 2}$ & $\sqrt{w d \sigma T}$ & $\sqrt{w d \sigma T} \times M^{-1 / 2}$ \\
SNR (III) & $w \sqrt{d \sigma T / B} \times M^{-1 / 2}$ & $w \sqrt{d \sigma T / B}$ & $w \sqrt{d \sigma T / B} \times M^{-1 / 2}$
\end{tabular}

$w$ energy density of the spectrum, $B$ of the background radiation, both per $\mathrm{cm}^{-1}$; $d \sigma$ limit of resolution and $\Delta \sigma$ spectral bandwidth in $\mathrm{cm}^{-1} ; M$ number of spectral elements $\Delta \sigma / d \sigma$. Luminosity and properties of each detector element are supposed to be identical between the three systems.

A practical example of each class is: a FTS, a grating spectrograph + Reticon array, a grating spectrograph working in scanner. The noise sources which are encountered in any observations give respectively condition I, II, III, when one of them becomes the dominant noise origin: (I) detector noise, (II) source photon noise, (III) thermal background noise. The table is made under the simple following assumptions: instruments have same luminosity, cover the same spectral range $\Delta \sigma$, work at the same resolution $\mathrm{d} \sigma$, in the same observing time. From this table, it can be concluded that a multiplex device has no advantages under condition I by comparison with a multichannel device, and becomes definitively slow with respect the same system under condition II and III. With the progress on photoelectric detectors, lowering detector noise to fundamental limits, and the general use of large telescopes, increasing available energy from the sources, condition I becomes less and less valid. The FTS is loosing its multiplex gain by a factor $\sqrt{M}$, with the event of large-size detector arrays, where $M$ is the number of spectral elements related to the number of pixels by the sampling factor. In other words, a grating spectrometer equipped with a low readout-noise $\mathrm{CCD}$, or an infrared array, has a fundamental gain in SNR with respect a FTS. But before to draw an abrupt conclusion, all the instrumental parameters which determine the SNR in real instruments: luminosity, resolution, spectral range, have to be carefully 
compared, which can radically change the conclusions. A FTS can remain an uncomparable tool for high-SNR spectroscopy.

\section{INSTRUMENTAL CHARACTERISTICS OF A FTS}

The factors which determine the signal-to-noise ratio in FT spectrum are reviewed to show the advantages of the method they contain:

\section{Resolving power}

The four currently working astronomical FTS (Davis et al 1980, Maillard and Michel 1982, Brault 1979, Hall et al 1979) have a maximum path difference respectively of $50,60,100,140 \mathrm{~cm}$. The limit of resolution in a spectrum depending only on the maximum path difference reached in the corresponding interferogram, low (few hundred) to very high $\left(10^{5}\right.$ or more) resolution can be continously selected. The resolving power can be tailored to the scientific purpose, providing the source is bright enough for the observing time available.

It is on the side of high resolution that a FTS is difficult to beat. In many cases, comparison of SNR as done in the previous paragraph, is without meaning because the grating spectrometer able to reach resolving powers of $10^{5}$ or more does not exist. This situation is particularly true in the infrared. Such a resolution corresponds to scanning over a path difference of $10 \mathrm{~cm}$ only at $2 \mu \mathrm{m}$ and $25 \mathrm{~cm}$ at $5 \mu \mathrm{m}$, which is much less than the limits offered by existing FTS. Similar resolutions would require gratings, if working at theoretical resolution, 50 $\mathrm{cm}$ wide at least,

The lack of resolution can be compensated by the association of a FabryPerot with a moderate resolution grating spectrometer, which is working as a monochromator (Wade 1983), or with a circular variable filter (Tanaka et al 1985). These systems are able to reach $3 \times 10^{4}$, (at the best), but suffer of a limited spectral coverage and a lack of versatility in changing of spectral range. Several etalons have to be used to cover for instance the $3-5 \mu \mathrm{m}$ region. It is better adapted to the study of the same line profile (as $\mathrm{B} \alpha$ or $\mathrm{B} \gamma$ ) in different objects (Persson et al 1984).

\section{Spectral range}

Change of spectral range with a FTS is easy. Equipped with InSb detectors, any domain limited by a filter, within the range $0.9-5.5 \mu \mathrm{m}$ can be selected. Two beamsplitters are enough to optimize the optical efficiency over such an extended range. On the CFH instrument (Maillard and Michel 1982), three beamsplitters (an additional one for the visible) are permanently installed in the instrument, mechanically mounted in such a way that the interchanging does not require any further adjustment. A choice of broad and narrow band filters within the range of sensitivity of the detectors, mounted on a 10-filter wheel, determines the width of the spectrum.

\section{Throughput}

From the classical relationship between the resolution $\mathrm{R}$ of an interferometric spectrometer and the solid angle of the entering beam, an equation can be deduced when the interferometer is matched to the throughput of a telescope of diameter $\mathrm{D}_{T}$, for a source of angular diameter :

$$
R=\frac{8}{\alpha^{2}}\left(\frac{D_{I}}{D_{T}}\right)^{2}
$$


The instrumental parameter which enters in the equation $D_{I}$ is the diameter of the parallel beam in the instrument. Numerical applications of this equation shows that for $\mathrm{D}_{I}=20 \mathrm{~mm}$, an extended source of $10^{\prime \prime}$ in diameter can be observed up to a resolving power of $10^{5}$. With a $8-\mathrm{m}$ telescope a beam size of $45 \mathrm{~mm}$ is enough to accept the same field at the same resolution. Lower resolutions allow bigger fields, usable for galactic nuclei, planetary nebulea, comets etc...In stellar applications, field-of-view does not seem to be so important. However, a spectrometer must be able to accept the whole seeing disk to reach the maximum luminosity. With modern 4-m class telescopes the luminosity of grating spectrographs becomes seeing-dependent for resolutions higher than $10^{4}$. In the ESO coude echelle spectrograph for instance, a slit width of 1 " has to be used for a resolution of $8 \times 10^{4}$. With this slit width, the transmission is equal to $65 \%$ with a 1 " (FWHM) seeing and falls to $35 \%$ with $2 "$ (Diego 1985). The luminosity drops dramatically for higher resolution.

\section{Substraction of parasitic background}

All the modern FTS systems are based on a design which employs cat's-eye retroreflectors. Two equal entrance apertures on the sky are matched on the two output detectors, allowing an automatic substraction of the parasitic sky background. The latter can be of thermal origin, above $2.5 \mu \mathrm{m}$, or parasitic daylight. This property is part of the photometric quality of FT spectra, particularly essential in infrared. That makes possible also to work in some cases in presence of daylight, which would be completly impossible with grating spectrographs. However, the energy of the source within the filter bandwidth must be brighter than the contribution of the sky in order to get the same SNR than in dark time. By respecting this condition, the multiplex "disavantage" offers the advantage of longer observing time.

\section{Metrologic accuracy}

In spectra obtained from a FTS, all the line positions are automatically calibrated to an absolute accuracy which can be equal to the accuracy to which the reference line of the instrument is known (generally a stabilized laser, $\Delta \lambda / \lambda \simeq 510^{-9}$ ). This property does not contribute to the SNR of a spectrum but takes advantage of high SNR. The accuracy of relative frequency determination is SNR limited. This unique quality, which is fully utilized in high-resolution, high-SNR laboratory spectroscopy, has also many advantages in astronomical applications. The identification of lines, the detection of new molecular species through several lines covering different quantum number, thanks to the large spectral coverage, can be made without ambiguity in crowdy spectra, providing accurate frequencies are also known in laboratory. This property has made the success of FT spectra. Many examples can be find in the reviews cited above. Radial velocities can be also easily measured and small lineshifts of $\simeq 100 \mathrm{~m} / \mathrm{s}$ detected (Maillard and Nadeau 1988).

In conclusion, this type of instrument is particularly suitable for all astronomical projects which benefit the most of all these characteristics: high resolution, large spectral range, accurate frequency calibration, eventually non-stellar angular diameter. The solar photosphere, the cool stellar atmospheres, the circumstellar envelopes, the dense molecular clouds, the planetary atmospheres, all objects with numerous, narrow lines are the fields where the FTS brings an essential contribution. Also, on faint emission-line objects, for which the multiplex 


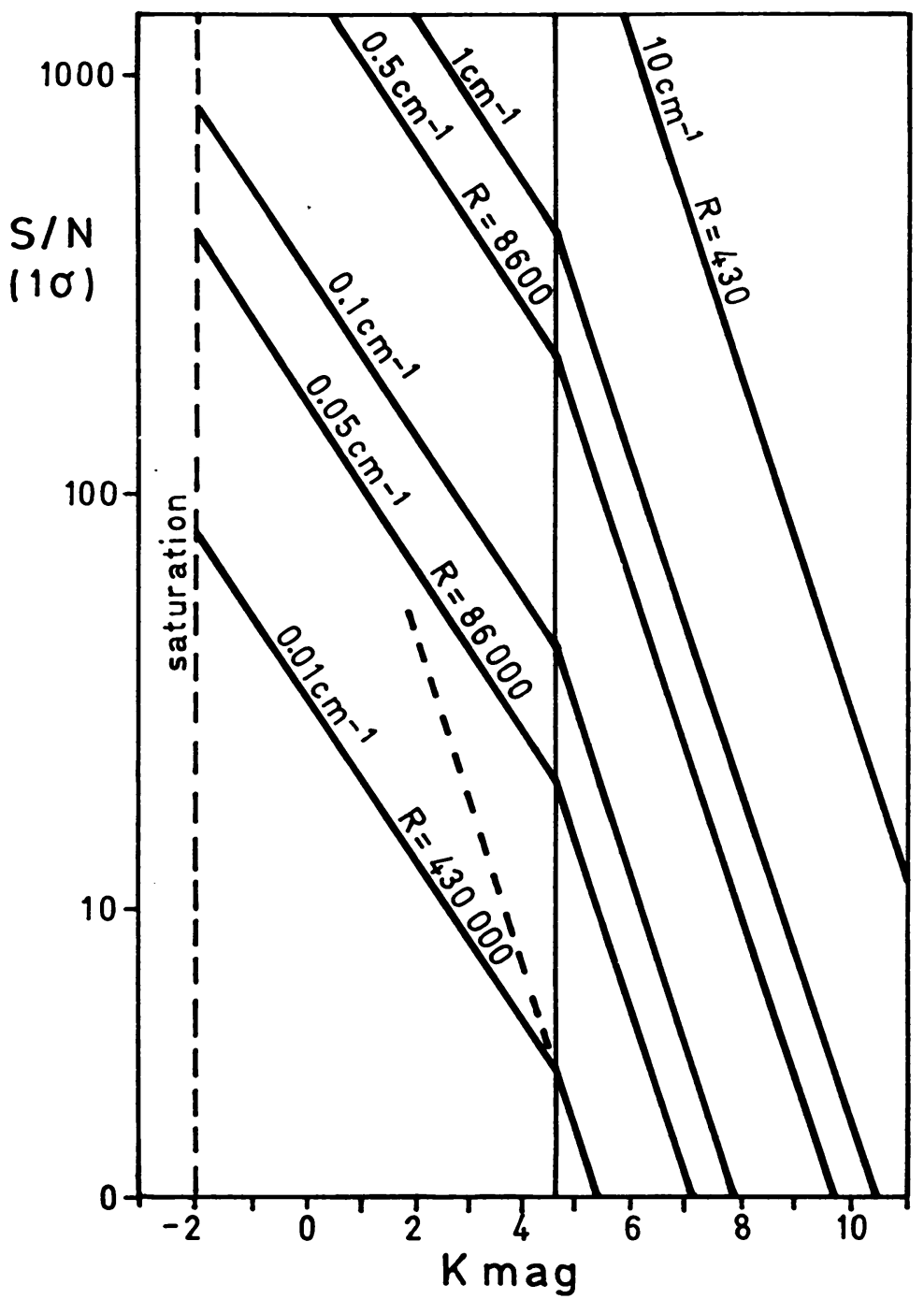

Fig. 1 Signal-to-noise ratio $(1 \sigma)$ versus $K$ magnitude for different resolution and 1 hour integration, with the CFH-FTS and a narrow band cold filter (peak $2.92 \mu \mathrm{m}, 0.11 \mu \mathrm{m}$ BWHM). The faster slopes correspond to detector noise limited conditions. Extrapolating those lines indicates by which factor SNR is deteriorated when photon source noise limited. For sources fainter than $K=4.6$ (vertical line) the full gain multiplex is recovered. At a limit of resolution of $.1 \mathrm{~cm}^{-1}$ such spectra contain more that 2000 spectral elements. 
gain remains almost intact, a FTS offers the advantage of a complete survey over an extraordinary large spectral range.

\section{IMPROVEMENT OF SNR WITH A FTS}

Optimization is possible by analyzing the factors which command the SNR in a FT spectrum. All the actions will tend to maximazise the signal and lower the photon noise to approach the detector noise limit.

- telescope size: as a FTS can accept a large throughput, it will be always more efficient behind a large telescope. Under condition I, SNR goes like $\mathrm{D}^{2}$ and like $\mathrm{D}$ under condition II and III. For high-resolution grating spectrograph, it varies only as $\approx \mathrm{D}$ and $\approx \sqrt{D}$ for comparable noise conditions.

- telescope plus FTS transmission: careful design with a minimum of optical pieces and selected beamsplitters can produce an instrument with a $70 \%$ optical efficiency against $30 \%$ for the best grating spectrograph. A high-resolution FTS can be compact enough to be installed at the cassegrain focus (Davis et al 1982 , Maillard and Michel 1982), providing a gain of a factor $\approx 1.5$ in luminosity with respect a coudé focus. If in addition, the infrared focus is used, as for the CFH-FTS, a significant gain is obtained in the thermal infrared by the low emissivity and the low temperature at this focus. Table II gives the final gain in SNR in comparison with a coudé system.

\section{TABLE II}

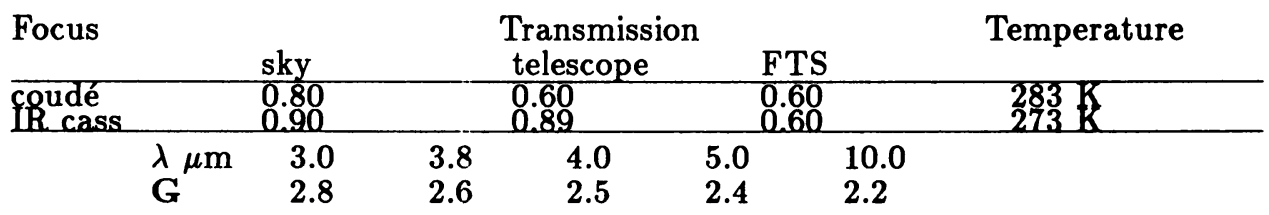

Gain $(G)$ in signal-to-noise ratio in the thermal infrared obtained by installing the FTS at the IR cassegrain focus of the CFH Telescope with respect a similar instrument in coudé, in a low elevation site. Emissivities and temperatures are lowered.

- spectral range: limiting the recorded spectral range to the useful range is an absolute rule to obtain the best SNR. A modern FTS has to be equipped with a large choice of narrow band filters. With the CFH-FTS and a narrow band filter in the CO 2-0 band (peak $2.32 \mu \mathrm{m}$ BWHM $0.11 \mu \mathrm{m}$ ), the system becomes detector noise limited for sources fainter than $\mathrm{K}=4.6$.In this range of brightness, the FTS benefits totally of the multiplex gain and there is no reason to use narrower filters.

- throughput: under condition III, an improvement is obtained also by limiting the cold aperture on the sky. SNR varies as $\alpha$. Against a widespread opinion, high-image quality in infrared is required, even with a FTS. With the CFH telescope, known for good image quality, diaphragms of 2.5 " are currently used in the FTS, which insured a $87 \%$ transmission for 1 " seeing (Diego 1985). In case a better seeing occurs (0.8"), 1.5" can be used, making an extra gain of a factor $\approx 1.7$.

- instrument thermal background: the background radiation is the sum of three contributions: the sky, the telescope and the instrument. The respective importances of the three terms for the CFH-FTS are: $0.053,0.066,0.4$, (from the parameters of Table II) which makes the instrument the dominant source of 
thermal background. Cooling the instrument to $-30^{\circ} \mathrm{C}$ would produce a reduction of a factor $\sim 2.5$ of the level of background radiation at $4.8 \mu \mathrm{m}$.

- association with array detectors: the development of array detectors can be a new chance for astronomical FTS.

In presence of thermal background, if a cold grating spectrograph is installed on one of the FTS outputs, it disperses the interferogram on the array detector. Each pixel can approach non-background limited conditions. A large spectral coverage, with all the other characteristics of a FTS, can be obtained, depending on the number of pixels. This option is particularly attractive in the $10 \mu \mathrm{m}$ window, at the maximum of thermal background. A group of Goddard Space Flight Center has already built a single helium-cooled post-disperser (Jennings et al 1986), to use on FTS facilities, on which a 20-element Si: As BIB array detector will be tested. A $7 \mathrm{~cm}^{-1}$ spectral range at $10 \mu \mathrm{m}$ will be covered near noise detector limited conditions.

In the visible and the near infrared, in presence of source photon noise, a similar device is the only way of reducing enough the flux received by each individual detector. Such a FTS system could combine the optimum SNR with the spectral coverage, regaining the full multiplex gain of a FTS.

Another use of array detectors associated with a FTS can be considered to perform 2D-spectroscopy on extended object. Using the throughput advantage and the stigmatic image in the plane of the detectors, interferograms from each pixel are recorded in parallel, corresponding to each point of the object. The amount of data to process has prevented developments in this direction. A project of FTS imager has been submitted anyway in view of the Space Telescope Program (Hall 1986).

\section{CONCLUSION}

Under observing conditions for which a FTS is not detector noise limited, it does not reach the ultimate SNR which would be possible to obtain with an ideal instrument, able to keep the detectors in these noise conditions. The lack of any other alternatives for scientific programs requiring in particular large spectral coverage and high resolution, imposes to accept this limitation. On the other hand, when efforts are made, in particular in the thermal infrared, significant gains in SNR can be obtained, which have been reviewed. Beyond all the improvements already tested with single detectors, a decisive step can be reached with a future generation of FTS systems associating array detectors. Then, all the characteristics of a FTS in resolution, spectral coverage could be exploited from the visible to the thermal infrared. The mixing of very different observing projects in spectral range and resolution, which is already possible during a telescope run, because any time is lost due to Aextensive change, could be completly achieved. With all the others, this factor should be kept in mind for the future instrumentation of coming very large telescopes.

References:

de Bergh, C., 6th International Conference on Fourier Transform Spectroscopy, Vienna 24 - 28 Aug 1987; proceedings to be published in Micro Chemica Acta ( Springer Verlag Pub. Co.).

Brault, J.W., 1979, Ossni. Mem. Oss. Astofis. Arcetri, 106, 33.

Davis, D.S., Larson, H.P., Williams, M., Michel, G., Connes, P., 1980, Applied Optics, 19, 4138. 
Diego, F., 1985, Pub. A.S.P., 97, 1209.

Hall, D.N.B., Ridgway, S.T., Bell, E.A., Yarborough, J.M., 1979, SPIE, 172, Instrumentation in Astronomy III, p.121.

Hall, D.N.B., 1986, Imaging Michelson Spectrometer for Space Telescope, U. of Hawaii Institut for Astronomy, Jet Propulsion Laboratory.

Jennings, D.E., Deming, D., Wiedemann, G.R., Kedy, J.J., 1986, Ap. J. (Letters), 310, L39

Maillard, J.P. and Michel, G., 1982, in Instrumentation for Astronomy with Large Optical Telescopes, IAU Colloquium No 67, C. M. Humphries (ed) p. 213, D. Reidel Pub. Co.

Maillard, J.P. and Nadeau, D., 1988, in The Impact of very high S/N Spectroscopy on Stellar Physics, IAU Colloquium No 132, Meudon 29 June - 3 July 1987.

Persson, S.E., Geballe, T.R., McGregor, P.J., Edwards, S., Lonsdale, C.J., 1983, Ap. J., 286, 289.

Ridgway, S.T. and Brault, J.W., 1984, Ann. Rev. Astrophys., 22, 291.

Tanaka, M., Yamashita, T., Satos, S., Okuda, H., 1985, Pub. A.S.P., 97, 1209.

Wade, R., 1983, SPIE, ㄴ5, 47.

\section{Discussion}

P.E Nissen: One advantage of the FTS, which you did not mention, is its clean spectral profile and negligible amount of scattered light. This makes the FTS an interesting instrument in connection with accurate determinations of profiles of spectral lines at a resolution $R 200,000$, to be used in e. g. the study of stellar atmospheric velocity fields.

J.P. Maillard: That's right. However, in this talk I wanted to focus on the instrumental characteristics of a FTS which directly enter in the determination of the $\mathrm{S} / \mathrm{N}$. These parameters have to be selected carefully to balance between the best $\mathrm{S} / \mathrm{N}$ to get and the advantages they offer. A clear example is the spectral range which has to be limited but gives access to many lines to analyze. The clean spectral profile is a supplementary quality but in the example you give a FTS would be required first for its capabilities of very high resolution.

J.E. Beckman: Could you please give us the result of your determination of D:H ratio on Mars?

J.P. Maillard: This ratio was measured with the CFH-FTS in Jan. 87 from the detection of the molecule HDO, at $3.7 \mu \mathrm{m}$, for the first time on Mars. An enhancement of this ratio by a factor 6 with respect to the terrestrial value was deduced, giving a new clue to the past history of water on Mars. I gave this example to illustrate the increased power of a FTS installed in a dry infrared site like Mauna Kea, at the focus of a low background telescope like CFHT. The large throughput, to accept the planetary disk, and the high resolution of the instrument were fully exploited. 